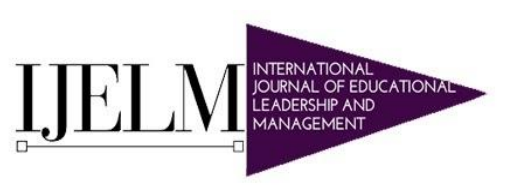

Hipatia Press

www.hipatiapress.com



Instructions for authors, subscriptions and further details:

http://ijelm.hipatiapress.com

\title{
Autopercepción del liderazgo docente
}

Jessica Sánchez-González ${ }^{1}$, Ana-Inés Renta-Davids ${ }^{1}$ y Juana-María Tierno-García ${ }^{1}$

1) Universitat Rovira i Virgili, Spain

Date of publication: January $16^{\text {th }}, 2022$

Edition period: January 2021 - July 2021

To cite this article: Sánchez-González, J., Renta-Davids, A.I., \& TiernoGarcía, J.M. (2022). Autopercepción del liderazgo docente. International Journal of Educational Leadership and Management. 10 (1), 84-109 doi: 10.17583/ijelm.2022.9597

To link this article: http://dx.doi.org/10.17583/ijelm.2022.9597

\section{PLEASE SCROLL DOWN FOR ARTICLE}

The terms and conditions of use are related to the Open Journal System and to Creative Commons Attribution License (CCAL). 


\title{
IJELM - International Journal of Educational Leadership and Management Vol 10 No. 1 January 2022
}

\section{Autopercepción del liderazgo docente}

\author{
Jessica Sánchez-González \\ Universitat Rovira i Virgili \\ Spain
}

\author{
Ana-Inés Renta-Davids \\ Universitat Rovira i Virgili \\ Spain
}

\author{
Juana-María Tierno-García \\ Universitat Rovira i Virgili \\ Spain
}

\section{Resumen}

El liderazgo es un factor clave en el éxito de un centro educativo. Este es hoy en día uno de los temas principales de la investigación educativa, sobre todo por lo que refiere a la dirección escolar. No obstante, es necesario determinar cuál es el papel de los docentes. Por tanto, la presente investigación pretende conocer y analizar la percepción docente en relación a la capacidad de liderazgo en el desarrollo de su actividad. Para dar respuesta, se ha llevado a cabo un estudio exploratorio con el que, por un lado, describir y categorizar el estilo de liderazgo ejercido, identificándolo como uno más autoritario o, por el contrario, uno más adaptativo. Y, por otro lado, se busca tratar de determinar la relación existente entre el estilo de liderazgo y las características sociodemográficas de la persona que lo ejerce. Para ello, se ha realizado un estudio de carácter cuantitativo, utilizando el cuestionario como instrumento de recogida de datos. De esta forma, se ha conocido el testimonio de 676 docentes de diferentes centros educativos de toda Cataluña. Los resultados derivados de la investigación muestran que los docentes se identifican con el liderazgo adaptativo, un estilo de movilizar que difiere de la exclusividad y conlleva adaptarse a las diferentes situaciones con el fin de ofrecer la respuesta educativa que más se ajuste a las necesidades de cada momento.

Palabras clave: liderazgo educativo, liderazgo intermedio, liderazgo autoritario, liderazgo adaptativo. 


\title{
Self-perception of teacher leadership
}

\author{
Jessica Sánchez-González \\ Universitat Rovira i Virgili \\ Spain
}

\author{
Ana-Inés Renta-Davids \\ Universitat Rovira i Virgili \\ Spain
}

\author{
Juana-María Tierno-García \\ Universitat Rovira i Virgili \\ Spain
}

\begin{abstract}
Leadership is a key factor in the success of an educational center. This is nowadays one of the main topics of educational research, especially in regard to school management. However, it is also necessary to determinate what the role of teachers is. Therefore, the present research aims to know and analyse the teaching perception in relation to the leadership capacity in the development of their activity. To give an answer, an exploratory study has been carried out which, on the one hand, describe and categorize the leadership style, identifying it as a more authoritarian or, on the opposite, a more adaptive one. On the other hand, it seeks to determine the relationship between the leadership style and the socio-demographic characteristics of whom exercises it. To this end, a quantitative study has been carried out, using a questionnaire as a data collection tool. In this way, the responses of 676 teachers from different educational centers throughout Catalonia were collected. The research results show that teachers identify with adaptive leadership, a style of mobilisation that differs from exclusivity and involves adapting to different situations in order to provide the educational response that best suits the needs of that time.
\end{abstract}

Keywords: educational leadership, middle-leadership, authoritarian leadership, adaptive leadership 
a situación actual de la educación plantea un gran reto y son muchas las dudas que han surgido y siguen surgiendo respecto a esta; aspectos como el fracaso escolar, el cambio constante de leyes educativas, la diversidad de modelos educativos, entre otros, son evidencias de esta situación de desconcierto.

En esta misma dirección, el modelo educativo en Cataluña se rige por un currículo cuyo contenido presenta una nueva perspectiva orientada al desarrollo competencial del alumnado. Este se desarrolla en base a la Ley de Educación (2009), que tiene como propósito hacer posible que la acción educativa se desarrolle en un marco que estimule la innovación y consolide las buenas prácticas. De esta forma, pretende responder a los cambios acelerados a los que se ve sometida la sociedad actual, a los contextos de una diversidad y una complejidad más acentuadas, a la necesidad de responder con inmediatez a las nuevas demandas que se explicitan y a los nuevos requerimientos sociales.

Sobre estas premisas, la Ley de Educación (2009) propone un conjunto normativo coherente que, entre otros aspectos, potencia la innovación pedagógica sistemática y estructurada y el reconocimiento de buenas prácticas educativas con el fomento y apoyo del liderazgo educativo.

El currículo en Cataluña comprende, para cada una de las enseñanzas del sistema educativo y de sus etapas, los objetivos, los contenidos, los métodos pedagógicos y los criterios de evaluación. También incluye las competencias básicas que se convierten en objetivos de etapa, los cuales surgen de las diferentes áreas curriculares.

Con respecto al ejercicio de la función docente, el profesorado es el responsable del proceso educativo. Asimismo, para afrontar esta situación de cambio en educación, es de gran importancia el perfil del educador. De sus actuaciones dependerá, en gran parte, la posibilidad de dejar atrás los paradigmas tradicionales basados en la memorización y repetición y aplicar los cambios necesarios para mejorar la situación actual y futura de la educación, optando por modelos más constructivistas basados en el desarrollo del propio conocimiento y el aprendizaje significativo. Por tanto, el rol del profesorado en el aula es, junto con la metodología, uno de los factores más importantes para promover en el alumnado la motivación por el conocimiento y la mejora de sus resultados académicos (Bush, 2021). 
Una de las acciones de las que forma parte la actuación docente, y que define en parte su perfil como educador, es el liderazgo. Este concepto se define como la relación de influencia entre líderes y seguidores que se proponen cambios y/o acciones que reflejan sus propósitos. Esta relación no se basa en el poder, sino que la comunicación entre los agentes implicados es horizontal (Rost, 1993).

Lo cierto es que el liderazgo es un concepto que cada vez adquiere más fuerza en el ámbito educativo. Especialmente, a raíz de los cambios inherentes a la situación derivada de la covid-19, que recientemente han "sacudido" a la comunidad educativa y han instado a que esta se adapte y se transforme en un tiempo récord, para poder hacerle frente (Serrano-Prato, 2020). No obstante, es necesario entender este concepto utilizado en infinitos campos y situaciones, no como la tarea de una persona específica, sino como una capacidad que cualquier agente de la comunidad educativa puede ejercer en diferentes escenarios. Los vínculos entre liderazgo y contexto se reconocen cada vez más, ya que el contexto influye fuertemente en el desarrollo del liderazgo. Al mismo tiempo, el contexto se ve influido por la cultura y, esta, a su vez, origina que el liderazgo se entienda y se lleve a cabo de forma diferente en función de las normas culturales (Bush, 2020).

En el siguiente marco teórico, se expone la evolución del concepto de "líder" a "capacidad de liderazgo", al mismo tiempo que se muestran dos estilos de liderazgo contrapuestos, como son el liderazgo autoritario y el liderazgo adaptativo.

\section{Fundamentación teórica}

Uno de los puntos clave para que el cambio y la transformación educativa se conviertan en una realidad y no se queden en teorías, es el papel y las acciones llevadas a cabo por los docentes en el proceso de enseñanza y aprendizaje. Si comparamos el rol que adoptaba el profesorado en el paradigma tradicional con los que se dan en las aulas actualmente, nos damos cuenta de que, en algunos casos, no existen grandes diferencias. A pesar de los avances en cuanto a las teorías sobre la enseñanza y el aprendizaje, se sigue actuando de forma muy similar. El docente actúa como una persona con la autoridad y el poder respecto al grupo, y es quien dice lo que se ha de realizar 
y quien evalúa las acciones del alumnado, que se limita a seguir las pautas marcadas. En los escenarios escolares, todavía operan los esquemas tradicionales, de forma que en pleno siglo XXI encontramos a alumnos que asumen un rol simplemente receptivo y docentes con el rol de reproducir los contenidos del libro, sin tener en cuenta los procesos de aprendizaje (Mendoza, 2011).

En la actualidad, el docente dispone de dos alternativas: por un lado, seguir con la más tradicional, basada en el almacenamiento de contenidos independientemente de si son significativos o no, que garantiza la seguridad instructora del docente, y, por otra parte, una alternativa basada en un aprendizaje más profundo que genere en el alumnado mecanismos de pensamiento más complejos, incluso metacognitivos. Aunque la segunda alternativa puede generar incertidumbre e inseguridad en el profesorado, ya que siempre se han seguido los mismos patrones de actuación, es necesario romper con los esquemas tradicionales y actuar con coherencia en relación con la demanda de la sociedad de cambio continuo (Blanco, 2010).

Para afrontar esta situación cambiante que presentan nuestra sociedad y el ámbito educativo, es necesario un perfil de docente innovador, alejado del perfil clásico basado en la imposición y la transmisión mecánica de conocimientos. El contexto actual de cambio nos pide elaborar una visión y definir nuevas estrategias que difieran del aprendizaje por repetición y la memorización de contenidos sin establecer relaciones entre ellos, automatizando hábitos de comportamiento tradicionalmente aceptados en sociedad (Torres, 2010).

Del mismo modo, se requiere un cambio en el perfil del docente, que se adecue a la situación actual, ya que no podemos emprender nuevas estrategias, metodologías, actuaciones, para afrontar las nuevas perspectivas siguiendo el perfil de actuación docente tradicional. Según Arends (2007), un docente debe caracterizarse, sobre todo, por: su compromiso con el aprendizaje, su capacidad de establecer relaciones humanas auténticas y una aptitud para crear un ambiente de aula socialmente justo y democrático.

Debido a la pertenencia a un modelo educativo basado en competencias, el perfil docente que se requiere actualmente en Cataluña es el de un profesional competente (Bolívar, 2011; Iranzo-García et al., 2018). Este concepto no tiene una definición clara y puede abordarse desde diferentes perspectivas, según el área implicada. Aunque el conjunto de profesionales pertenecientes a las 
diferentes áreas que forman parte del Currículum de Educación, coincide en que un docente competente es aquel que: tiene un dominio amplio de la ciencia referente (1), es un buen comunicador (2), sabe cómo, cuándo y por qué debe poner estos conocimientos al servicio del alumnado (3), orienta y acompaña al alumnado en el proceso de enseñanza-aprendizaje (4), propicia situaciones que potencian el desarrollo de las capacidades individuales y sociales del alumnado (5) y da un valor a la competencia de autonomía personal y aprender a aprender (6). De modo que un docente competente es aquel que sabe formar ciudadanos libres, capaces de tener unos criterios y de desarrollarse con éxito dentro de la sociedad de la que forman parte (Salvat et al., 2002).

\section{El concepto de líder}

A lo largo de la historia, han sido muchos los autores que han descrito el rol de los "líderes" como grandes personas con una posición de superioridad frente al resto. Pero, lógicamente, ha habido una evolución en el concepto desde que Platón mostró en la República que los filósofos más preparados debían regir la sociedad, hasta autores más cercanos como Cifuentes-Medina et al. (2020).

En un inicio, se creía que el "líder" poseía unas capacidades innatas que le capacitaban para actuar como tal, hasta que a principios del siglo XX se cuestionó si realmente "líder" se nacía o se hacía durante la vida, y se propuso que la situación y el contexto eran los factores que llevaban a determinadas personas al frente de los grupos (Barrientos, 2021). Asimismo, CifuentesMedina (2020) refuerza esta idea y define el liderazgo como una conducta humana que surge naturalmente y por instinto, de la misma forma que en la época primitiva la organización en manadas y el surgimiento de un "líder" se daba por supervivencia.

"Líder" proviene del término inglés "lead", cuyo significado es guiar, dirigir, mandar, encabezar (como verbo) y mando, dirección, delantera (como sustantivo). La persona que actualmente se llama "líder" ha sido y es aquella que guía y dirige a un equipo de trabajo a asumir retos que emergen de forma acelerada por los cambios vertiginosos a los que se enfrentan los centros educativos día a día (Rodríguez Revelo, 2017). 


\section{La capacidad de liderazgo}

El concepto de "líder" como una persona con unas características innatas en un primer momento y de una persona que se convertía en "líder" en función de la situación y el contexto, evolucionó hacia el concepto de liderazgo, de modo que el foco de atención se desplaza de las capacidades de la persona, hacia las conductas y las acciones que se llevaban a cabo. Aunque en un primer momento el liderazgo se asoció a una visión individualista con las funciones de medir y supervisar, más tarde ha adquirido un carácter más participativo, derivando hacia una perspectiva de liderazgo múltiple y compartido (Bolívar,2010; Gros et al. 2013). Asimismo, son muchos los autores que han avalado la capacidad de liderazgo como factor clave para el éxito y transformación educativa (Day, 2017; Bolívar, 2019; Barrero et al., 2020; Ceballos, 2021).

Esta capacidad de liderazgo se ha atribuido históricamente a los líderes escolares, a quién se les ha otorgado la responsabilidad de generar unos determinados resultados en la escuela (Bolívar, 2017). No obstante, cada vez se hace más evidente la necesidad de unos líderes intermedios que den soporte a la dirección (Searby et al., 2016; Bonilla et al., 2020). De esta manera se propone distribuir este liderazgo entre los líderes educativos y el profesorado (Harris, 2014), derivando hacia una perspectiva de liderazgo múltiple y compartido (Bolívar, 2010).

Cifuentes-Medina (2020) apunta que el liderazgo está presente en todas las etapas de la vida y, en consecuencia, no todo líder ni todo estilo de liderazgo son válidos para determinados ambientes. Por este motivo, y gracias a las diferentes conceptualizaciones que han realizado los autores, se han definido muchos estilos de liderazgo, los cuales se pueden clasificar de la siguiente forma: liderazgo autoritario y liderazgo adaptativo.

\section{El liderazgo autoritario}

El liderazgo autoritario se basa en el control y la imposición mediante el desarrollo de la autoridad por parte del docente (Mendoza, 2011). Este estilo de liderazgo se adecua a los paradigmas tradicionales de la educación, basados en la memorización y repetición de contenidos, en los que los docentes toman el protagonismo del proceso de enseñanza aprendizaje, actuando como transmisores de información. En efecto, el liderazgo autoritario comprende la autonomía en la toma de decisiones, dirigiendo y controlando equipos. Entre 
sus principales rasgos característicos destacan: administración de premios y castigos al alumnado en función de su comportamiento (1), imposición de normas de conducta en las actividades académicas (2) y mantenimiento del orden y respeto con autoridad durante sus clases (3) (Villegas Yarleque et al., 2021).

Por consiguiente, los seguidores son motivados por sus intereses personales, en lugar de ser influidos por su líder para trascender sus propias necesidades en beneficio de la organización (Castro y Lupano, 2005). Otros autores como Villa Sánchez (2019) definen este estilo como liderazgo transaccional, que suele darse cuando los líderes premian o sancionan, dependiendo de si el rendimiento de los seguidores es el esperado. De esta forma podemos decir que se basa en una transacción o proceso de intercambio entre el líder y sus seguidores, donde se satisfacen las necesidades de los subordinados a cambio de que se cumplan los objetivos planteados. Este está configurado por dos factores: la recompensa contingencial y la dirección por excepción.

\section{El liderazgo adaptativo}

El liderazgo adaptativo es un proceso de movilización de un grupo para que este afronte una realidad incierta y desarrolle nuevas capacidades que le permitan asegurar su progreso y bienestar. Para ello, trata de movilizar al grupo, no de darle respuestas, sino de conseguir que este sea consciente de que existe una realidad incierta la cual requiere el desarrollo de nuevas capacidades (Martin, 2009).

En concreto, Heifetz (1997) lo caracteriza de la siguiente forma:

- No hay un líder, sino una persona que ejerce liderazgo, por lo que se contrapone a la visión tradicional, de la persona con superioridad frente al grupo.

- Se basa en movilizar trabajo adaptativo, concepto que consiste en orientar y focalizar la atención, apoyando a quienes afrontan un reto que genera desequilibrio, para que sean estas personas quienes busquen las soluciones y restablezcan su propio equilibrio.

- Todo el mundo puede ejercer el liderazgo en una situación determinada. 
En definitiva, el liderazgo adaptativo sitúa el énfasis en el comportamiento de la persona que ejerce el liderazgo y no en la persona en sí. A diferencia del liderazgo autoritario, las personas que ejercen el liderazgo adaptativo no se basan en el control y la imposición, sino en la capacidad de transmitir confianza, respeto y crear motivaciones mediante la comunicación. Esta perspectiva considera que el liderazgo no es cuestión de unas personas privilegiadas, sino que es un comportamiento que cualquier persona puede llevar a cabo.

Según Blanco (2010), la capacidad de liderar es la habilidad de visionar el mundo y convencer a los demás para que busquen confiadamente y con entusiasmo la consecución de los objetivos y refuercen positivamente el patrimonio emocional de las personas con grandes aspiraciones. Villegas Yarleque et al. (2021) refuerzan esta idea, aunque denominan a este liderazgo democrático, ya que el líder consulta con los seguidores analizando opiniones $\mathrm{y}$, consecuentemente, tomando decisiones.

Por tanto, el liderazgo adaptativo en el ámbito educativo puede equipararse al liderazgo transformacional, concepto empleado por diferentes autores en el ámbito empresarial y organizativo. De acuerdo con Villa Sánchez (2019), el liderazgo transformacional se da cuando los líderes amplían y elevan los intereses de sus seguidores, cuando generan conciencia y aceptación de los propósitos y la misión del grupo, y cuando motivan a sus seguidores a ver más allá de sus propios intereses por el bien de los demás. El estilo de liderazgo expuesto implica el incremento de las capacidades de los miembros de la organización para resolver individual o colectivamente los diferentes problemas y lo que se entiende como la toma de decisiones.

\section{Método}

\section{Enfoque metodológico de la investigación}

La presente investigación se ha desarrollado como un estudio exploratorio de carácter cuantitativo, cuyo objetivo es conocer, analizar y describir la autopercepción docente con respecto al estilo de liderazgo ejercido. De este objetivo se desprenden las siguientes preguntas de investigación: 
1. ¿Cómo varía el estilo de liderazgo ejercido en función de las características sociodemográficas de la persona que lo ejerce (Edad, género, nivel de estudios, etapa en la que se imparte docencia y la titularidad del centro en que trabaja)?

2. ¿Con qué estilo de liderazgo se identifica el profesorado en el desarrollo de su actividad docente?

3. ¿Cómo influye el liderazgo percibido por el profesorado durante su etapa formativa en el liderazgo ejercido durante su ejercicio docente? ¿Se reproducen los mismos patrones o se adoptan nuevos?

Para ello, se ha diseñado un cuestionario y se ha distribuido de forma telemática a todos los centros educativos de Cataluña mediante el correo corporativo del centro. De este modo, hemos obtenido 676 respuestas.

\section{Participantes}

La muestra del estudio está formada por 676 docentes en activo, de los cuales el 76,2\% son mujeres. Los participantes tienen una edad media de 43,81 años y el 66,1\% tiene más de 11 años de experiencia en la docencia. El 89,9\% de los docentes trabaja en centros públicos y el 73,5\% imparte clases en Educación Primaria o Educación Secundaria. La tabla 1 expone con más detalle los datos sociodemográficos de la muestra.

Tabla 1. Contextualización de los docentes a partir de los datos del cuestionario

\begin{tabular}{lccc}
\hline & Min-Max & M & DS \\
\hline Edad (años) & $23-66$ & $\mathbf{4 3 , 8 1}$ & $\mathbf{1 0 , 1 6 7}$ \\
\hline & & Frecuencia & Porcentaje \\
\hline
\end{tabular}

\section{Género}

Mujer 
Año de finalización de estudios

M

DS

$1975-1984$

41

154

$1985-1994$

$1995-2004$

$2005-2014$

$2015-2021$

Valores perdidos

Nivel de estudios

Ciclos formativos

Grado/Licenciatura/Diplomatura

Estudios superiores

Postgrado

Máster

Doctorado

Valores perdidos

\section{Años de experiencia en docencia}

Menos de 1 año

De 1 a 5 años

6 a 10 años

11 a 20 años

Más de 21 años
3

0,45

576

85,2

5

0,75

9

1,3

68

10,1

13

1,9

2

0,3
6,1

22,8

29,7

24,1

14,5

2,8 
Etapa educativa en la que imparte docencia

Educación Infantil

Educación Primaria

Educación Secundaria Obligatoria

Bachillerato

Ciclos Formativos

Universidad

Formación de adultos

Otros

\section{Titularidad del centro educativo}

Centro público

Concertado / Privado

Total
M

74

263

234

10

40

4

20

31
4,6

DS

10,9

38,9

34,6

1,5

6

0,6

2,9

*M (Media), DS (Desviación estándar)

\section{Instrumento}

El cuestionario está organizado en dos partes. Una primera parte recoge información sociodemográfica tales como la edad, el género, el nivel de estudios, el año de promoción, los años de experiencia en docencia y la etapa en la que trabajan en la actualidad los docentes encuestados. La segunda parte recoge información sobre las variables analíticas del estudio a través de preguntas cerradas sobre la propia percepción en relación al liderazgo ejercido como docentes, así como también al liderazgo percibido en el profesorado durante su etapa como alumnos. En la tabla 2 se exponen las preguntas incluidas en el cuestionario. 
Tabla 2. Variables descriptivas analizadas en el cuestionario implementado.

\begin{tabular}{lc} 
Variables descriptivas & Escala de respuesta \\
\hline Edad & Respuesta abierta \\
Género & Mujer - Hombre - Otros \\
Nivel de estudios & Respuesta abierta \\
Año de promoción & Respuesta abierta \\
Años de experiencia en docencia & Menos de 1 año - Entre 2 y 5 años - Entre 6 y 10 años \\
Etapa en la que imparten docencia & $\begin{array}{c}\text { Educación Infantil - Educación Primaria - Educación } \\
\text { Secundaria Obligatoria - Bachillerato - Ciclos } \\
\text { Formativos - Universidad - Formación de adultos - } \\
\text { Ontros }\end{array}$
\end{tabular}

Pregunta

Opciones de respuesta

Liderazgo ejercido

\begin{tabular}{|c|c|c|c|c|}
\hline $\begin{array}{c}\text { ¿Qué estilo de } \\
\text { liderazgo } \\
\text { consideras que } \\
\text { adoptas en el } \\
\text { desarrollo de tu } \\
\text { práctica docente? }\end{array}$ & Autoritario & Adaptativo & $\begin{array}{l}\text { Combinación } \\
\text { de los dos, } \\
\text { predominante } \\
\text { adaptativo }\end{array}$ & $\begin{array}{l}\text { Combinación } \\
\text { de los dos, } \\
\text { predominante } \\
\text { autoritario }\end{array}$ \\
\hline
\end{tabular}

\section{Liderazgo percibido}

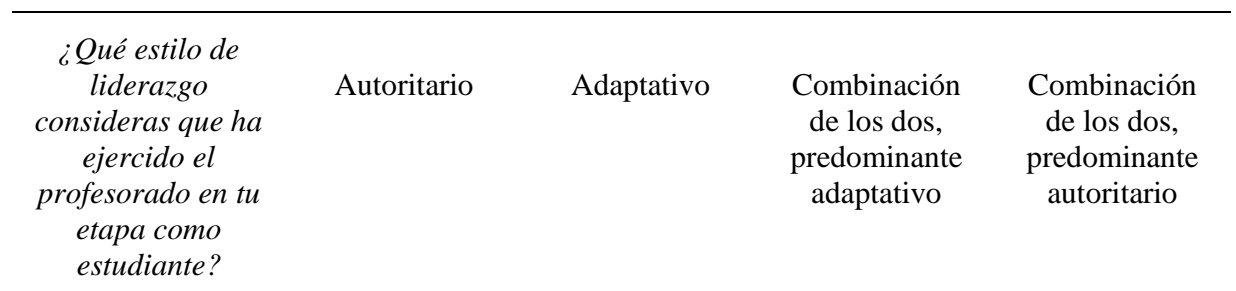




\section{Análisis de datos}

En primer lugar, se ha realizado un análisis estadístico descriptivo de las variables para caracterizar la muestra analizada. En segundo lugar, se ha aplicado un análisis estadístico inferencial a través de tablas cruzadas entre las variables sociodemográficas y las variables analíticas relacionadas con el estilo de liderazgo percibido y el estilo de liderazgo ejercido. En cada caso, se ha calculado el coeficiente chi-cuadrado para determinar la independencia de las variables.

\section{Resultados}

A continuación, se presentan los principales resultados obtenidos atendiendo a los objetivos planteados en el diseño de la investigación.

\section{a) ¿Con qué estilo de liderazgo se identifica el profesorado en el desarrollo de su actividad docente?}

La mayoría de los docentes de la muestra perciben el liderazgo ejercido como una combinación del liderazgo autoritario y el adaptativo, aunque con predominancia del adaptativo $(48,8 \%)$, mientras que una proporción un poco más reducida se identifican con el ejercicio de un liderazgo completamente adaptativo (37,6\%). Una proporción reducida de la muestra percibe el ejercicio de su liderazgo como una combinación de ambos con predominancia autoritaria $(13,3 \%)$ y la proporción de los docentes que perciben el liderazgo autoritario es residual $(0,3 \%)$.

Tabla 3. Percepción docente en relación al estilo de liderazgo ejercido.

Frecuencia

Porcentaje

\section{Estilo de liderazgo}

Adaptativo

254

37,6

Autoritario

2

0,3

Combinación de los dos, predominante adaptativo

330

48,8

Combinación de los dos, predominante autoritario

90

13,3

Total

676

100 


\section{b) ¿Cómo varía el estilo de liderazgo ejercido en función de las características sociodemográficas de la persona que lo ejerce?}

En primer lugar, no se ha identificado influencia de la edad sobre el estilo de liderazgo ejercido. Así lo evidencia la prueba de chi-cuadrado, la cual sitúa su valor en 5,883 ( $\mathrm{p}>0.05$ ). Por lo tanto, la edad no condiciona la percepción del liderazgo ejercido por los docentes.

Tabla 4. Tabla cruzada entres las variables edad y estilo de liderazgo.

\begin{tabular}{llcccc}
\hline & & \multicolumn{3}{c}{ Edad (Agrupada en intervalos) } & \\
& & $\mathbf{2 3 - 3 9}$ & $\mathbf{4 0}-\mathbf{5 6}$ & $\mathbf{5 7 - \mathbf { 6 6 }}$ & Total \\
\hline $\begin{array}{l}\text { Tipo } \\
\boldsymbol{d e} \\
\text { liderazgo }\end{array}$ & Adaptativo & 87 & 85 & 77 & 249 \\
& $\begin{array}{l}\text { Autoritario } \\
\text { Combinación de los dos, } \\
\text { predominante adaptativo } \\
\text { Combinación de los dos, } \\
\text { predominante autoritario }\end{array}$ & 113 & 120 & 92 & 325 \\
\hline Total & 22 & 34 & 34 & 90 \\
\hline
\end{tabular}

*Valores perdidos: 10

En segundo lugar, con relación a la influencia del género en la adopción de uno u otro estilo de liderazgo, los resultados muestran que existe una relación significativa entre dichas variables $(\mathrm{x} 2=20,573, \mathrm{p}<0.001)$. Se aprecia una mayor proporción de hombres con tendencia a percibir un estilo de liderazgo adaptativo, mientras que las mujeres tienden a percibir un liderazgo combinado con predominio del estilo adaptativo. Esto indica que el género puede tener una influencia en la percepción del tipo de liderazgo ejercido.

Tabla 5. Tabla cruzada entre las variables género y estilo de liderazgo.

Género

Mujer Hombre Otros

Total 


\begin{tabular}{|c|c|c|c|c|c|c|c|c|c|}
\hline & & $\mathbf{F}$ & $\%$ & $\mathbf{F}$ & $\%$ & $\mathbf{F}$ & $\%$ & $\mathbf{F}$ & $\%$ \\
\hline \multirow{4}{*}{$\begin{array}{l}\text { Tipo de } \\
\text { liderazgo }\end{array}$} & Adaptativo & 193 & 37,5 & 61 & 38,6 & 0 & 0 & 254 & 37,6 \\
\hline & Autoritario & 1 & 0,2 & 1 & 0,6 & 0 & 0 & 2 & 0,3 \\
\hline & $\begin{array}{l}\text { Combinación de los dos, } \\
\text { predominante adaptativo }\end{array}$ & 255 & 49,5 & 75 & 47,5 & 0 & 0 & 330 & 48,8 \\
\hline & $\begin{array}{l}\text { Combinación de los dos, } \\
\text { predominante autoritario }\end{array}$ & 66 & 12,8 & 21 & 13,3 & 3 & 100 & 90 & 13,3 \\
\hline Total & & 515 & 100 & 158 & 100 & 3 & 100 & 676 & 100 \\
\hline
\end{tabular}

En tercer lugar, con relación al tipo de estudios cursados, tampoco se observa ninguna relación entre esta variable y el estilo de liderazgo ejercido por el docente $(x 2=11,762, \mathrm{p}>0.05)$.

Tabla 6. Tabla cruzada entre el tipo de estudios de los docentes y el liderazgo ejercido.

Tipo de liderazgo ejercido

Total

$\begin{array}{ccc}\text { Adaptativo Autoritario } & \begin{array}{c}\text { Combinación } \\ \text { de los dos, } \\ \text { predominante } \\ \text { adaptativo }\end{array} & \begin{array}{c}\text { Combinación } \\ \text { de los dos, } \\ \text { predominante } \\ \text { autoritario }\end{array}\end{array}$

\begin{tabular}{|c|c|c|c|c|c|c|}
\hline \multirow[t]{6}{*}{$\begin{array}{l}\text { Tipo } \\
\text { de } \\
\text { estudios }\end{array}$} & $\begin{array}{l}\text { Grado/ } \\
\text { Licenciatura/ } \\
\text { Diplomatura }\end{array}$ & 214 & 2 & 281 & 79 & 576 \\
\hline & Postgrado & 3 & 0 & 6 & 0 & 9 \\
\hline & Máster & 23 & 0 & 36 & 9 & 68 \\
\hline & Doctorado & 10 & 0 & 2 & 1 & 13 \\
\hline & $\begin{array}{l}\text { Ciclo } \\
\text { formativo }\end{array}$ & 3 & 0 & 4 & 1 & 8 \\
\hline & Perdidos & 1 & 0 & 1 & 0 & 2 \\
\hline Total & & 254 & 2 & 330 & 90 & 676 \\
\hline
\end{tabular}


En cuarto lugar, los resultados muestran que la etapa en la que se imparte docencia no está asociada de forma significativa al estilo de liderazgo percibido $(x 2=37,18, p>0.05$. Este se lleva a cabo indistintamente de la edad a la que se dirige la enseñanza.

Tabla 7. Tabla cruzada entre la etapa en la que se imparte docencia y el estilo de liderazgo desarrollado.

Tipo de liderazgo ejercido

Total

\begin{tabular}{|c|c|c|c|}
\hline Adaptativo & Autoritario & $\begin{array}{l}\text { Combinación } \\
\text { de los dos, } \\
\text { predominante } \\
\text { adaptativo }\end{array}$ & $\begin{array}{l}\text { Combinación } \\
\text { de los dos, } \\
\text { predominante } \\
\text { autoritario }\end{array}$ \\
\hline
\end{tabular}



\begin{tabular}{llcccccccccc}
\hline Etapa & E.I. & 37 & 14,6 & 0 & 0 & 32 & 9,7 & 5 & 5,5 & 74 & 10,9 \\
& E.P. & 95 & 37,4 & 0 & 0 & 135 & 40,9 & 33 & 36,8 & 263 & 38,9 \\
& E.S.O. & 71 & 27,9 & 2 & 100 & 115 & 34,9 & 46 & 51,1 & 234 & 34,6 \\
& Bach & 4 & 1,6 & 0 & 0 & 6 & 1,8 & 0 & 0 & 10 & 1,5 \\
& C.F. & 16 & 6,3 & 0 & 0 & 19 & 5,8 & 5 & 5,5 & 40 & 5,9 \\
& Univ & 3 & 1,2 & 0 & 0 & 0 & 0 & 1 & 1,1 & 4 & 0,6 \\
& Adult & 11 & 4,3 & 0 & 0 & 9 & 2,7 & 0 & 0 & 20 & 3 \\
& Otros & 17 & 6,7 & 0 & 0 & 14 & 4,2 & 0 & 0 & 31 & 4,6 \\
\hline Total & & 254 & 100 & 2 & 100 & 330 & 100 & 90 & 100 & 676 & 100 \\
\hline
\end{tabular}

*Educación Infantil (E.I.), Educación Primaria (E.P), Educación Secundaria (ES), Bachillerato (Bach), Ciclos Formativos (C.F.), Universidad (Univ), Formación de adultos (Adult), Otros.

En último lugar, la titularidad del centro no determina la percepción del estilo de liderazgo ejercido. Así lo indica el coeficiente chi-cuadrado, que sitúa su valor en $1,347(\mathrm{p}>0.05)$. 
Tabla 8. Tabla cruzada entre la titularidad del centro en el que se encuentra el docente y el ejercicio de liderazgo.

\begin{tabular}{llccc}
\hline & & \multicolumn{2}{c}{ Titularidad } & Total \\
& & $\begin{array}{c}\text { Concertado / } \\
\text { Privado }\end{array}$ & $\begin{array}{c}\text { Centro } \\
\text { público }\end{array}$ & \\
\hline $\begin{array}{l}\text { Tipo } \\
\text { de }\end{array}$ & Adaptativo & 22 & 232 & 254 \\
& Autoritario & 0 & 2 & 2 \\
& $\begin{array}{l}\text { Combinación de los dos, } \\
\text { predominante adaptativo }\end{array}$ & 35 & 295 & 330 \\
& $\begin{array}{l}\text { Combinación de los dos, } \\
\text { predominante autoritario }\end{array}$ & 11 & 79 & 90 \\
\hline & & 68 & 608 & 676 \\
\hline
\end{tabular}

c) ¿Cómo influye el liderazgo percibido por el profesorado durante su etapa formativa, en el liderazgo ejercido durante su ejercicio docente? ¿Se adquieren y reproducen los mismos patrones o se adoptan nuevos?

Se observa un gran cambio en la percepción que tienen los docentes con relación al liderazgo percibido durante la etapa formativa y el liderazgo ejercido como docentes. Más de la mitad de la muestra analizada, 58,4\%, considera que durante su etapa formativa el profesorado ejerció sobre ellos un estilo de liderazgo autoritario, mientras que este valor queda reducido a un $0,3 \%$ cuando se les pregunta sobre el estilo de liderazgo propio ejercido como docente. En la misma línea, un $28 \%$ de los docentes indica que durante su etapa formativa el profesorado ejercía el liderazgo como combinación de los dos estilos con predominancia del autoritario, y este porcentaje queda reducido a un $13,3 \%$ de los participantes que perciben el ejercicio de su liderazgo de este modo. Por el contrario, solo un 6,1\% percibió el liderazgo durante su etapa formativa como adaptativo en contraste con el 37,6\% de los 
docentes que lo perciben como adaptativo. Lo mismo ocurre con la combinación de los dos estilos de liderazgo con preponderancia adaptativa, en donde se observa un $7,5 \%$ de docentes que lo percibieron durante su etapa formativa y un $48,8 \%$ de docentes que lo perciben durante su ejercicio docente.

Tabla 9. Tabla cruzada entre la percepción docente del estilo de liderazgo ejercido y el estilo de liderazgo percibido en su etapa como alumnos/as.

\begin{tabular}{|c|c|c|c|c|}
\hline & \multicolumn{2}{|c|}{$\begin{array}{c}\text { Liderazgo } \\
\text { ejercido (como } \\
\text { docente) }\end{array}$} & \multicolumn{2}{|c|}{$\begin{array}{c}\text { Liderazgo } \\
\text { percibido (en su } \\
\text { etapa formativa }\end{array}$} \\
\hline & Frec. & $\%$ & Frec. & $\%$ \\
\hline Adaptativo & 254 & 37,6 & 41 & 6,1 \\
\hline Autoritario & 2 & 0,3 & 395 & 58,4 \\
\hline $\begin{array}{l}\text { Combinación de los dos, predominante } \\
\text { adaptativo }\end{array}$ & 330 & 48,8 & 51 & 7,5 \\
\hline $\begin{array}{l}\text { Combinación de los dos, predominante } \\
\text { autoritario }\end{array}$ & 90 & 13,3 & 189 & 28,0 \\
\hline Total & 676 & 100 & 676 & 100 \\
\hline
\end{tabular}

\section{Discusión}

Los resultados obtenidos apuntan a un gran cambio entre el liderazgo percibido por el profesorado en su formación inicial y el liderazgo ejercido en su etapa como docentes. Esto puede explicarse gracias a la evolución que ha experimentado en nuestra sociedad del conocimiento el concepto de liderazgo, concretamente el liderazgo intermedio. Diversos autores como Uribe et al (2016) evidencian que el nivel intermedio del liderazgo es central para la implementación de cualquier reforma o cambio en el territorio, así como para enfrentar los desafíos inherentes. 
La mayor parte del profesorado considera que los docentes con los que estuvo en contacto en su etapa formativa ejercían un liderazgo autoritario. No obstante, consideran no haber reproducido este patrón e identifican el liderazgo ejercido en el desarrollo de su rol como un liderazgo adaptativo. Los resultados muestran cómo, tanto hombres como mujeres, se identifican más con estilos predominantemente adaptativos. Este hecho resulta de gran importancia debido a que se ajusta al liderazgo descrito por Heifetz (1997), un estilo en el que el docente-líder moviliza trabajo adaptativo.

Alvarado-Macías y Zambrano-Zambrano (2021), en su estudio sobre el liderazgo transformacional y su influencia en los equipos de negocio del sector financiero, muestran cómo el liderazgo transformacional es ejercido en un porcentaje mayor por el género masculino, con respecto al femenino. Así lo muestran nuestros resultados, ya que las mujeres se han identificado mayormente con un estilo que combina los enfoques autoritario y adaptativo, aunque con ligero predominio de este último. Abello-Romero et al. (2019) refuerzan esta idea con su estudio en el que demuestran cómo los factores relacionados con el contexto y las características personales influyen en la preferencia por un tipo de liderazgo. No obstante, en el presente estudio no se ha podido observar dicha relación y únicamente se ha identificado una relación significativa respecto al género de la persona que desarrolla el liderazgo.

Teniendo en cuenta los objetivos de esta investigación, las hipótesis planteadas y el análisis realizado, hemos observado que factores sociodemográficos como la edad, el nivel de estudios, la etapa en la que se imparte docencia o el centro educativo, no influyen en el desarrollo de un determinado estilo de liderazgo. No obstante, la variable género sí que lo hace. De acuerdo con Arteaga y Ramón (2009), al género masculino tradicionalmente siempre se le han atribuido características competitivas, de control detallado, de razonamiento analítico y con el objetivo de triunfar en las tareas. En cambio, a las mujeres se les atribuyen características cooperativas, de empatía, menor control de la situación y orientación hacia la calidad de las relaciones. Esto podría explicar por qué las mujeres han descrito su liderazgo ejercido como una combinación del estilo autoritario y adaptativo, aunque con una mayor representación del adaptativo. Los hombres, en cambio, han mostrado ser más categóricos y se han definido como líderes adaptativos $(38,6 \%$ vs $37,5 \%)$. 
El liderazgo adaptativo se caracteriza por situar la importancia en el comportamiento de la persona que ejerce el liderazgo, y no en la persona en sí. Martin (2009) lo define como la capacidad de movilización de un grupo para que este afronte una realidad incierta y desarrolle nuevas capacidades que le permitan asegurar su progreso y bienestar. Por consiguiente, este planteamiento conlleva la adaptación del rol ejercido según el contexto al que se dirige. De ahí que la exclusividad no tiene por qué ser lo ideal, sino que es necesario adaptarse a las diferentes situaciones y ofrecer la respuesta educativa que más se ajuste a las necesidades de ese momento.

Esta valoración coincide con Rodríguez Revelo (2017), quien considera clave buscar la complementariedad entre los distintos estilos, aprovechando los aspectos positivos de cada uno. Por otro lado, aunque podemos extraer diferentes percepciones en cuanto a la predisposición al cambio, existe una dominancia de la buena predisposición.

Cabe reconocer que este estudio presenta algunas limitaciones que condicionan la interpretación de los resultados. Una muestra de ello son las preguntas con las que se busca conocer la percepción docente en relación a la capacidad de liderazgo, pues debido a su formulación pueden estar sujetas al factor de "deseabilidad social". Socialmente presenta connotaciones negativas ser un "líder" autoritario, este hecho puede condicionar a que prácticamente ningún miembro de la población estudiada se decante por las opciones que se asocian a este estilo de liderazgo. Esto podría explicar la relación con el hecho que ambos géneros se perciben mayoritariamente como líderes adaptativos. No obstante, no se dispone de suficientes datos como para establecer una relación causal entre el estilo de liderazgo descrito y el factor de "deseabilidad social".

\section{Conclusión}

El liderazgo docente es de gran importancia en el proceso de enseñanza y aprendizaje, siendo el segundo factor más influyente en el rendimiento del alumnado después de la metodología utilizada en el aula (Bush, 2021).

El objeto de estudio es de gran importancia para la práctica docente, sobre todo de cara a próximas situaciones de enseñanza y aprendizaje. Su trasfondo, con la práctica docente como eje vertebrador, proporciona una orientación con el estilo de liderazgo a realizar, con la intención de mejorar la 
actuación docente y consecuentemente el desarrollo competencial del alumnado.

Asimismo, surgen líneas futuras de investigación que proporcionen datos más concluyentes. En concreto, hacen falta estudios que se centren en la capacidad de liderazgo de los docentes y la influencia del contexto en esta. También es necesario profundizar en el papel que tiene el género para explicar las diferencias identificadas. ¿Es cierto que el género femenino tiende a estilos de liderazgo más adaptativos?, ¿Es el contexto el que determina este enfoque? $\mathrm{O}$, por el contrario, ¿Son los rasgos de personalidad los que determinan un estilo de liderazgo concreto, independientemente del género y del contexto en el que se encuentra?

\section{Referencias}

Abello-Romero, J., Mancilla, C., y Ganga-Contreras, F. (2019). Factores que influyen en la preferencia por el tipo de estructura de liderazgo del máximo cuerpo colegiado de universidades iberoamericanas. Revista de la Educación Superior, $\quad 48 \quad$ (190), 23-43. https://doi.org/10.36857/resu.2019.190.710

Alvarado-Macias, M., y Zambrano-Zambrano, E.J. (2021). El liderazgo transformacional y su influencia en los equipos de negocio del sector financiero del cantón Portoviejo. Digital Publisher, 6 (3), 423-441. https://doi.org/10.33386/593dp.2021.3.632

Arends, R.I. (2007). Aprender a enseñar. Mc-Graw-Hill.

Arteaga, A., y Ramón, S. (2009). Liderazgo resonante según género. Multiciencias, 9(3), 289-295

Barrero Fernández, B., Domingo Segovia, J., y Fernández Gálvez, J. D. (2020). Liderazgo intermedio y desarrollo de comunidades de práctica profesional: Lecciones emergentes de un estudio de caso. Psicoperspectivas, 19(1), 1-13. DOI:10.5027/psicoperspectivas-Vol19Issue1-fulltext-1751

Barrientos, R. (2021). El temperamento sí importa: Características psicológicas necesarias para liderar escuelas. Revista Educación y Sociedad, 2(3), 48-57. https://doi.org/10.53940/reys.v2i3.60

Blanco, P. (2010). Docents innovadors i estratègicament competents. Paper Kite. 
Bonilla-Murillo, E. y Solís-Herebia, V.S. (2020). El liderazgo docente: ¿Por qué es importante para el desarrollo de los estudiantes? Revista Dilemas Contemporáneos: Educación, Política y Valores, 9(3), 1-18. https://doi.org/10.46377/dilemas.v36i1.2307

Bolívar, A. (2019). Marco español para la dirección escolar e identidad profesional: contexto, desarrollo e implicaciones. Archivos Analíticos de Políticas Educativas, 27(114). https://doi.org/10.14507/epaa.27.4544

Bolívar, A. (2017). El liderazgo pedagógico de la dirección escolar en España: limitaciones y acciones. Licinio Lima y Virginio Sá (Orgs.). O Governo das escolas. Democracia, controlo e performatividade. Edições Humus, pp. 151-171

Bolívar, A. (2011). Aprender a liderar líderes. Competencias para un liderazgo directivo que promueva el liderazgo docente. Educar, 472, 253-275.

Bolívar, A. (2010). El liderazgo educativo y su papel en la mejora: Una revisión actual de sus posibles limitaciones. Psicoperspectivas, 9(2), 9-33. DOI:10.5027/PSICOPERSPECTIVAS-VOL9-ISSUE2-FULLTEXT-112

Bush, T. (2021). Assessing successful school leadership: ¿What do we know? Educational Management Administration \& Leadership, 49(5) 687-689. https://doi.org/10.1177/17411432211034675

Bush, T. (2020). School leadership and culture: Societal and organisational perspectives. Educational Management Administration \& Leadership, 49(2) 211-213. https://doi.org/10.1177/1741143220983063

Castro, A., y Lupano, M.L. (2005). Diferencias individuales en las teorías implícitas del liderazgo y la cultura organizacional percibida. Boletín de Psicología, 85, 89-109

Ceballos, N., y Saiz, Á. (2021). Autonomía escolar al servicio del éxito educativo para todos. Archivos Analíticos de Políticas Educativas, 29(22). https://doi.org/10.14507/epaa.29.5366

Cifuentes-Medina, J. E., González-Pulido, J. W., y González-Pulido, A. (2020). Efectos del liderazgo escolar en el aprendizaje. Panorama, 14(26), 78-93. https://doi.org/10.15765/pnrm.v14i26.1482

Day, C. (2017). School Leadership as an Influence on Teacher Quality. En: Zhu, X., Goodwin, A. y Zhang, H. (Eds.), Quality of teacher education and learning: Theory and practice (pp.101-117). Springer. https://doi.org/10.1007/978-981-10-3549-4_7

Gros, B., Fernández-Salinero, C., Martínez, M., y Roca, E. (2013). El liderazgo educativo en el contexto del centro escolar. En XXXII 
Seminario Interuniversitario de teoría de la educación. Liderazgo y Educación.

Harris, A. (2014). Distributed leadership matters: Perspectives, practicalities, and potential. Corwin.

Heifetz, R. (1977). Sin respuestas fáciles. Paidós.

Iranzo-García, P., Camarero-Figuerola, M., Barrios-Arós, C., Tierno-García, J.-M., y Gilabert-Medina, S. (2018). ¿Qué Opinan los Maestros sobre las Competencias de Liderazgo Escolar y sobre su Formación Inicial? REICE. Revista Iberoamericana Sobre Calidad, Eficacia Y Cambio En Educación, 16(3). https://doi.org/10.15366/reice2018.16.3.002

Ley 12/2009, del 10 de julio, de Educación. (2009). Boletin Oficial del Estado.

Legislación consolidada. https://boe.es/buscar/pdf/2009/BOE-A-200913038-consolidado.pdf

Martin, I. (2009). Liderazgo adaptativo y autoridad. Cátedra liderazgos y gobernanza democrática. 19, 6-49

Mendoza, D. (2011). El liderazgo educativo. Xihmai. 6, 7-20.

Rodríguez Revelo, E. (2017). Función directiva y estilos de liderazgo educativo: términos no sinonímicos, sino complementarios. Alternativas, 18(3), 5-18. http://dx.doi.org/10.23878/alternativas.v18i3.165

Rost, J.C. (1993). Leadership for the Twenty-First Century. Westport: Praeger. En Rosari, R. (2019) Leadership definitions application for lecturers' leadership development. Journal of Leadership in Organizations, 1(1), 17-28. https://doi.org/10.1177/107179199300100109 Salvat, A., Gavaldà, A., Gimeno, S., Pérez, M.C., Viscarro, I., Marqués, L., Macaya, A., Ollé, A., Aymí, J., Girondo, L., y Ríos, M. (2002). El mestre competent. Què hauria de posseir i què hauria de transmetre de les diverses àrees curriculars? Comunicació Educativa, 15, 3-9. https://doi.org/10.17345/comeduc20024-9

Searby, L. J., y Armstrong, D. (2016). Supporting the development and professional growth of middle space educational leaders through mentoring. International Journal of Mentoring and Coaching in Education, 5(3), 162-169. https://doi.org/10.1108/IJMCE-06-2016-0054

Serrano-Prato, G.P. (2020). Liderazgo Educativo en tiempos post pandemia. Aportes clave desde una mirada filosófica educativa. Saberes Andantes, 3(7), 48-68. https://doi.org/10.53387/sa.v3i7.58 
Torres, J. (2010). La justicia curricular. El caballo de Troya de la cultura escolar. Morata.

Uribe, M., Castillo, P., Berkowitz, D., y Galdames, S. (2016). 'Panorámica sobre el liderazgo y gestión local de educación. Lo que sabemos de la investigación internacional y la evidencia nacional'. Informe Técnico No.

4. LIDERES EDUCATIVOS, Centro de Liderazgo para la Mejora Escolar: Chile.

Villa Sánchez, A. (2019). Liderazgo: una clave para la innovación y el cambio educativo. Revista de Investigación Educativa, 37(2), 301-326. http://dx.doi.org/10.6018/rie.37.2.365461

Villegas Yarleque, M., Villegas Aguilar, G. F., Apaza Panca, C. M., Chinchay Villarreyes, S. S., y Lujan-Vera, P. E. (2021). Liderazgo del docente universitario y el rendimiento académico. Universidad Ciencia $Y$ Tecnología, 25(108), 30-37. https://doi.org/10.47460/uct.v25i108.428 
Jessica Sánchez-González, graduada en Educación Primaria por la Universidad Rovira i Virgili (URV). Actualmente, cursando el Máster de Innovación en la Intervención Social y Educativa en la URV. Asimismo, colabora con el Departamento de Pedagogía de la misma Universidad en líneas de investigación relacionadas con el liderazgo educativo y la mejora escolar.

Dirección de contacto: Universitat Rovira i Virgili. Facultat de Ciències de l'Educació i Psicologia. Departament de Pedagogia. Carretera de Valls, s/n. 43007 Tarragona

E-mail: jessica.sanchez@urv.cat

Ana-Inés Renta-Davids, profesora Serra Hunter en la Universitat Rovira i Virgili y miembro del grupo de investigación Metodología de la Investigación Educativa con Impacto Social (MEDIS). Sus principales líneas de investigación son el aprendizaje en el lugar de trabajo, la empleabilidad, el aprendizaje informal, el desarrollo de competencias y la evaluación de competencias, y la formación inicial y continua del profesorado.

Dirección de contacto: Universitat Rovira i Virgili. Facultat de Ciències de 1'Educació i Psicologia. Departament de Pedagogia. Carretera de Valls, s/n. 43007 Tarragona

E-mail: $\underline{\text { anaines.renta@urv.cat }}$

Juana María Tierno-García, profesora agregada (contratada doctora) del Departamento de Pedagogía la Universidad Rovira i Virgili (URV). Licenciada en Pedagogía y doctora por la URV. Principales líneas de investigación: evaluación educativa, docencia universitaria y dirección de centros educativos. Miembro de la Red de Excelencia de Investigación sobre Liderazgo y Mejora Educativa (RILME)

Dirección de contacto: Universitat Rovira i Virgili. Facultat de Ciències de l'Educació i Psicologia. Departament de Pedagogia. Carretera de Valls, s/n. 43007 Tarragona

E-mail: juanamaria.tierno@urv.cat 